\title{
Fractal Dimensions and Geometries of Caves ${ }^{1}$
}

\author{
Rane L. Curl ${ }^{2}$
}

Lengths of all caves in a region have been observed previously to be distributed hyperbolically, like self-similar geomorphic phenomena identified by Mandelbrot as exhibiting fractal geometry. Proper cave lengths exhibit a fractal dimension of about 1.4. These concepts are extended to other self-similar geometric properties of caves with the following consequences.

Length of a cave is defined as the sum of sizes of passage-filling, linked modular elements larger than the cave-defining modulus. If total length of all caves in a region is a self-similar fractal, it has a fractal dimension between 2 and 3; and the total number of linked modular elements in a region is a self-similar fractal of the same dimension. Cave volume in any modular element size range may be calculated from the distribution.

The expected conditional distribution of modular element sizes in a cave, given length and modulus, also is distributed hyperbolically. Data from Little Brush Creek Cave (Utah) agree and yield a fractal dimension of about 2.8 (like the Menger Sponge). The expected number of modular elements in a cave equals approximately the 0.9 power of length of the cave divided by modulus. This result yields an intriguing "parlor trick." An algorithm for estimating modular element sizes from survey data provides a means for further analysis of cave surveys.

KEY WORDS: caves, fractals, self-similarity.

\section{INTRODUCTION}

Lengths of all caves in each of a number of regions are distributed approximately hyperbolically (Pareto) (Curl, 1960, 1966). Smoothed cumulative length distributions for 10 geographic regions (Fig. 1) were estimated for all caves in each region, including those without entrances, from measured length distributions of caves with one or more entrances, using the procedure described by Curl (1966). Expressed mathematically, the number of caves longer than $l$ is given by

$$
N(l)=N\left(l_{0}\right)\left(\frac{l}{l_{0}}\right)^{-\nu}
$$

\footnotetext{
'Manuscript received 13 September 1985; accepted 10 April 1986.

${ }^{2}$ Department of Chemical Engineering. University of Michigan--Dow Building, Ann Arbor, Michigan 48109 .
} 


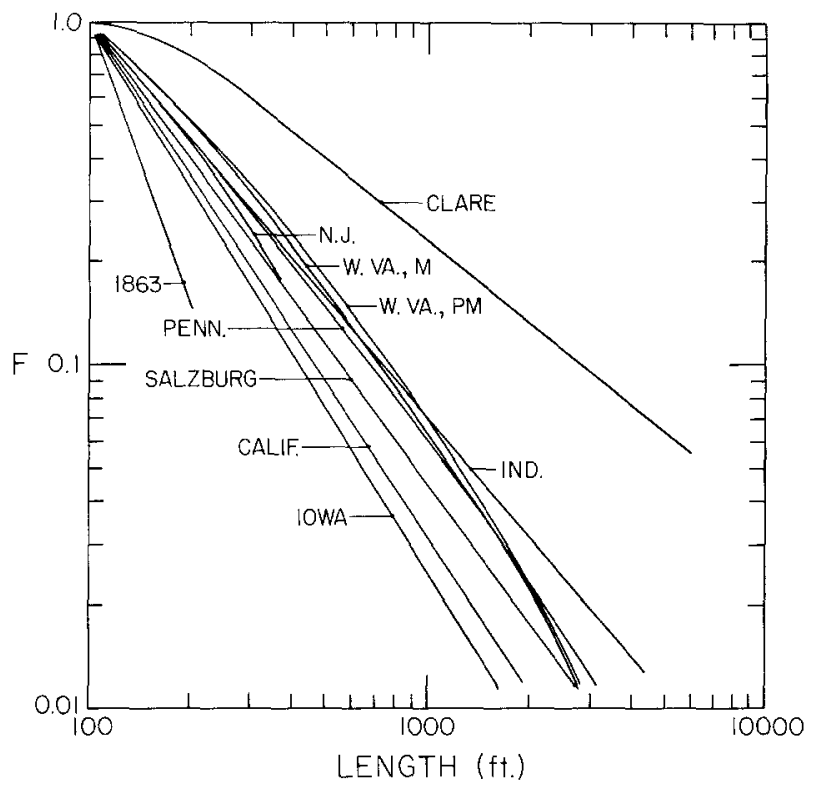

Fig. 1. Approximate (smoothed) cumulative fraction length distributions of all caves longer than $100 \mathrm{ft}$ in several regions. Retraced with permission from Curl (1966). Copyright 1966 by The University of Chicago. All rights reserved.

where $N\left(l_{0}\right)$ is number of caves longer than a reference length $l_{0}$. Except for curves labeled "1863" and "Clare" (Fig. 1), exponent $\nu$ lies between 1.2 and 1.6 .

Many geographic objects appear to exhibit a hyperbolic size distribution, at least over some range of sizes. Korčak (1940) and Fréchet (1941) first remarked upon this for areas of lakes, areas of islands, and lengths of rivers, and it has come to be called "Korčak's Law." A more modern example is sizes of lunar microcraters (Morrison and Clanton, 1979), which can be represented by eq. 1 over at least two decades of size, and hence six decades of mass.

Mandelbrot (1983) has shown that Korčak's Law is a consequence of fractal fragmentation, where a distribution of sizes of islands is produced by a class of (infinite) repetitive processes of subdivision of geometric figures. The exponent in eq. 1 is identified by Mandelbrot as fractal or similarity dimension of resulting geometric figures which are, usually but not necessarily, self-similar at every scale. This dimension is an extension of integer (topological) dimensions of length, area, or volume, and need not be an integer. The most important concept that will be borrowed from Mandelbrot is that natural objects are known that can be considered to exhibit fractal geometry with noninteger dimension. Within this context, extrapolate to $l=0$ (Fig. 1), where $N$ goes to infinity; we 
may consider the distribution of cave lengths as a natural fractal. Not illogically, an infinite number of caves of length zero or greater may exist.

Equation 1 has been shown also to be the consequence of a growth process in which caves grow at a rate proportional to their size but with the proportionality constant for growth rate arbitrarily but independently distributed (Curl, 1960). If the distribution ever has the form of eq. 1, it then will always have that form. The result was called an invariant growth population. This was offered as a possible explanation for occurrence of distributions shown (Fig. 1). Processes causing departures from hyperbolic distributions also were discussed.

A third interpretation of eq. 1 arises from the fact that all of its momentintegrals are undefined over the interval $0<l<\infty$. That is, a hyperbolically distributed quantity has no characteristic lengths. Conversely, if a phenomenon arises without any controlling characteristic lengths, it must be distributed hyperbolically. Of course, some characteristic lengths are bound to enter, but if they are important only outside a wide range of $l$, eq. 1 might be expected to apply over that range.

The fractal, growth, or characteristic-length interpretations of Fig. 1 and eq. 1 do not reveal directly any details about geomorphic processes responsible for the distribution of lengths of caves, but the distribution of lengths of caves does contain information about the geometry of caves and possibly constrains ideas about geomorphic processes. Fractal interpretation, in particular, permits some extrapolation of geometric concepts from available length data.

Difficult questions exist in defining cave geometry for purposes of geomorphic interpretation and comparison. A question as simple as how to define the length of a cave has not yet been settled (e.g., Chabert and Watson, 1981). Cave length data (Fig. 1) are based on survey lengths. These are total lengths of connected lines or "strings" that more-or-less follow paths taken by explorers. The basic geometric problem of attaching an unequivocal length to a threedimensional volume has not been resolved. A fractal approach is described here.

Although cave surveyors record cave passage shapes and show these on their maps, such three-dimensional data are seldom accurate enough (if available) to define cave "shape" quantitatively and, e.g., to permit calculating cave volume. The fractal approach is useful in putting some "flesh" on cave lengths to give volume to survey strings. Consequences of this include predictions about geometric properties of cave passages in accessible caves, as well as predictions about those that are too small for human exploration but which are important biologically, hydrologically, and in the study of cave origins.

\section{CAVE MODULUS}

Length of a cave admittedly is rather meaningless without specifying the size of the explorer. A cave is a subterranean volume that, in principle, can be explored and surveyed to obtain a length; it will be greater for a small explorer 
than a large explorer. In addition, a cave that can be explored by a given size explorer becomes fragmented into numerous caves by a larger explorer that cannot go between fragments. To define a cave, therefore, requires specification of shape and characteristic size of the explorer. If the standard explorer is chosen to be a sphere, diameter of that sphere is called the modulus, $\mu$, of the sodefined cave. If the explorer is human and the modulus is the size of a human, the cave is called a proper cave. Other explorer shapes could be chosen. These concepts and definitions are presented and discussed by Curl (1964).

Equation 1 is therefore a distribution conditional on modulus $\mu$ and should be written

$$
N(l \mid \mu)=N\left(l_{0} \mid \mu\right)\left(l / l_{0}\right)^{-\nu}
$$

Lengths of caves (Fig. 1) are defined by the proper modulus $\mu_{\mathrm{p}}$ or the length scale appropriate for a human explorer. A cave is defined as the envelope of volume that can be explored by a standard (spherical) explorer of modulus $\mu$ (Fig. 2).

Because modulus is a characteristic length, one would expect it to impose a characteristic length upon the hyperbolic distribution of eq. 2. In particular, eq. 2 must be truncated at small $l$ because cave length cannot be less than (approximately) diameter $\mu$ of the standard explorer. Mandelbrot (1983) calls this an inner cutoff. This is not contradictory to the previous statement about $N$ approaching infinity as $l \rightarrow 0$ because a finite explorer had not yet been defined. Figure 3 shows the smoothed distribution of $l$ for all proper caves of Pennsylvania (Curl, 1966). $l$ was truncated at $15.24 \mathrm{~m}$ or $50 \mathrm{ft}$, because few data were

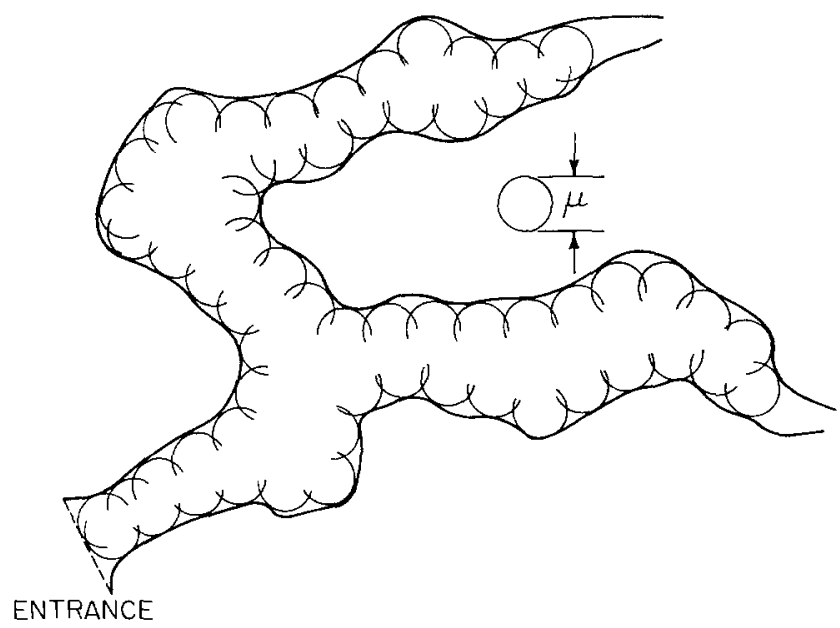

Fig. 2. Cave space defined by a specified modulus $\mu$. 


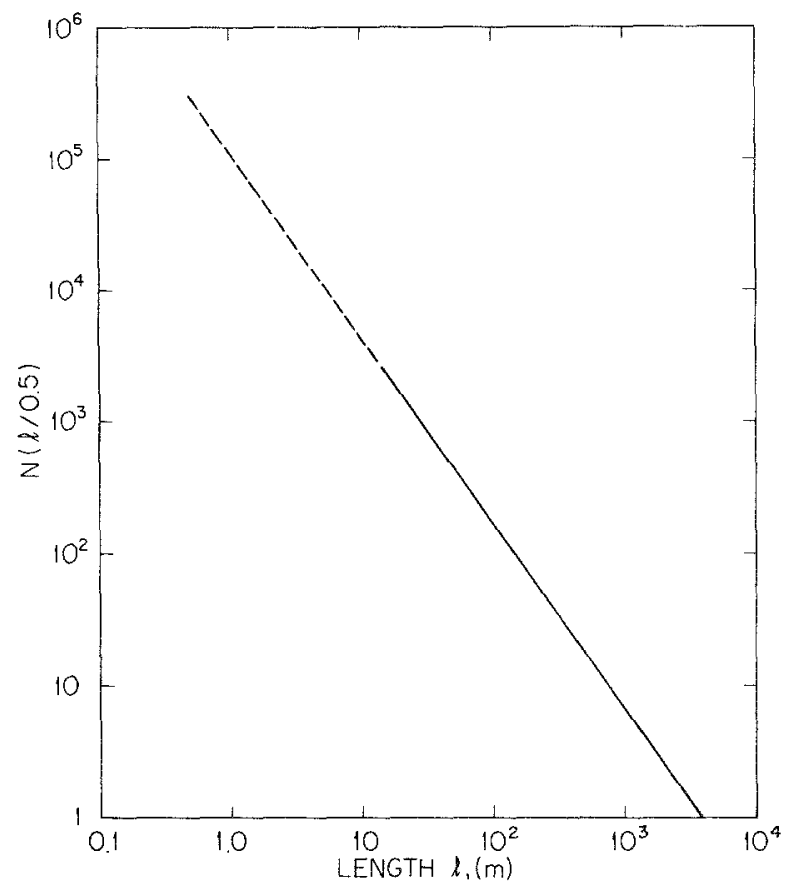

Fig. 3. Idealized distribution of proper cave lengths in Pennsylvania (Curl, 1966). $\nu=1.4$, the cutoff $l_{0}=15.24 \mathrm{~m}$, and extrapolation is to $\mu_{p}=0.5 \mathrm{~m}$.

reported for shorter caves, but the distribution is shown extrapolated to $l=$ $0.50 \mathrm{~m}$, a nominal proper modulus. Fractal (similarity) dimension is 1.4. The equation for the distribution line is

$$
N(l \mid 0.5)=2526\left(\frac{l}{15.24}\right)^{-\nu} \quad l>0.5 \mathrm{~m}
$$

Total number of all proper caves in Pennsylvania is $3.0 \mathrm{E}+05$ (setting $l=$ $0.50 \mathrm{~m}$ ). Most would be entranceless.

The number density $n(l \mid \mu)$ of all caves in a region of modulus $\mu$ is the negative derivative of eq. 2 with respect to $l$.

$$
\begin{array}{ll}
n(l \mid \mu)=-\frac{d N(l \mid \mu)}{d l}=\frac{\nu N\left(l_{0} \mid \mu\right)}{l_{0}}\left(\frac{l}{l_{0}}\right)^{-\nu-1} \quad \mu<l \\
n(l \mid \mu)=0 \quad l<\mu
\end{array}
$$

Total (sum) length of all caves of modulus $\mu$ in a region, with individual 
lengths between $l_{1}$ and $l_{2}\left(\mu<l_{1}\right)$, is given by

$$
\int_{l_{1}}^{l_{2}} \ln (l \mid \mu) d l=\frac{\nu l_{0}^{\nu} N\left(l_{0} \mid \mu\right)}{\nu-1}\left(l_{1}^{1-\nu}-l_{2}^{1-\nu}\right)
$$

The fractal interpretation would expect that length would go to infinity as $l_{1} \rightarrow 0$, which requires that $\nu$ be greater than 1.0 . In addition, total length may be infinite, but not plane-filling, and therefore fractal dimension should lie between 1 and 2. These interpretations are compatible with convergence in the limit $l_{2} \rightarrow \infty$, with $\nu>1$, which is valid if a finite volume of caves exists in a region, because the minimum cave diameter is $\mu$. Note that $1<\nu<2$ (Fig. 1) is in accord with fractal theory. In the limit $l_{1} \rightarrow 0$, eq. 5 diverges for $\nu>$ 1.0 , and an infinite cave volume would seem to be predicted, but eq. 4 is truncated for $l<\mu$. Again, no contradiction is found. Equation 4 is an allowable length density distribution for natural fractal of cave lengths.

Total (sum) length of all caves of modulus $\mu$ in a region is given by eq. 5 with $l_{1}=\mu$ and $l_{2} \rightarrow \infty$. This is

$$
L(\mu)=\frac{\nu l_{0}^{\nu} N\left(l_{0} \mid \mu\right)}{\nu-1} \mu^{1-\nu}
$$

Mean length $\bar{l}$ can be obtained by dividing eq. 6 by eq. 2 with $l=\mu$. For Pennsylvania, $L(0.5)=5.3 \mathrm{E}+05 \mathrm{~m}$. Mean length is $\vec{l}=1.8 \mathrm{~m}$.

\section{RICHARDSON'S LAW FOR CAVE LENGTHS}

Richardson (1961) observed that lengths of a number of geographic frontiers (boundaries) depended upon length of the "measuring stick" used. That is (in Richardson's words), "walking a pair of dividers along . . . the frontier, so as to count the number of equal sides of a polygon." The relation he found for total length of a frontier can be expressed by

$$
L(\eta)=\eta^{1-\beta}
$$

where $\eta$ is length of each side of the polygon. For the west coast of Britain, he found $\beta=1.25$, for $\eta$ from 10 to $1000 \mathrm{Km}$.

Equation 7 is also the relation for the length of a number of geometric figures generated by (infinite) reticulation of a line of unit length. Such a "fractal curve" is, for example, the Koch curve, for which $\beta=1.2618$. The Koch curve is self-similar at every scale and has, in the limit $\eta \rightarrow 0$, infinite length. Mandelbrot (1983) shows that this is also a geometric figure with a fractal dimension given by $\beta$.

As the defining modulus $\mu$ for caves in a region decreases, total length of all caves, $L(\mu)$, increases without limit. That is, as an explorer becomes smaller, more and more small passages could be surveyed. The relation would have to 
be monotonic in $\mu$, but whether it would follow eq. 7 is unknown. However, because cave lengths are distributed (approximately) according to eq. 2 , the assumption that eq. 7 describes total length of caves in a region as a function of cave modulus is not unreasonable. At the least, exploring consequences of making that assumption is interesting. Therefore, let

$$
\frac{L(\mu)}{L\left(\mu_{\mathrm{p}}\right)}=\left(\frac{\mu}{\mu_{\mathrm{p}}}\right)^{1-\beta}
$$

\section{MODULUS EFFECT UPON CAVE NUMBER}

Equation 8 can apply only to total cave length in a region, not to individual caves. Individual caves are extended as $\mu$ decreases, but some separate caves of a larger modulus would become connected at a smaller modulus. In addition, subterranean voids that did not exist as caves of a given modulus, because they were too small to admit a standard explorer of that modulus, could come into existence with a smaller modulus. Decreasing the modulus will both connect caves and create new caves. Whether the net effect will be to increase or decrease the total number of caves in a region can be ascertained.

Equation 6 for proper caves is

$$
L\left(\mu_{\mathrm{p}}\right)=\frac{\nu l_{0}^{\nu} N\left(l_{0} \mid \mu_{\mathrm{p}}\right)}{\nu-1} \mu_{\mathrm{p}}^{1-\nu}
$$

and the ratio of eq. 6 to eq. 9 , substituted into eq. 8 , gives

$$
\frac{N\left(l_{0} \mid \mu\right)}{N\left(l_{0} \mid \mu_{\mathrm{p}}\right)}=\left(\frac{\mu}{\mu_{\mathrm{p}}}\right)^{\nu-\beta}
$$

An assumption has been made that $\nu$ is independent of $\mu$. This may be reasonable because $\nu$ is an inherent fractal dimension of fragmentation and is dimensionless, whereas $\mu$ is an arbitrary exploration modulus with the dimension of length. Another fundamental characteristic length must exist for them to be related. None is known. A test is remotely possible if cave data permitted calculating surveys with an arbitrary modulus $\left(\mu>\mu_{\mathrm{p}}\right)$. This has not been done.

Equation 10 is the ratio for caves longer than $l_{0}$ in a region of number of caves of modulus $\mu$ to number of proper caves. The question of whether decreasing $\mu$ increases or decreases the number of caves is a question of whether $\nu$ is smaller or larger than $\beta$.

The process by which caves are both connected and created as $\mu$ decreases or both fragmented and lost as $\mu$ increases is shown (Fig. 4) for three examples. Here, increasing $\mu$ increases, does not affect, or decreases the number of cave fragments. 

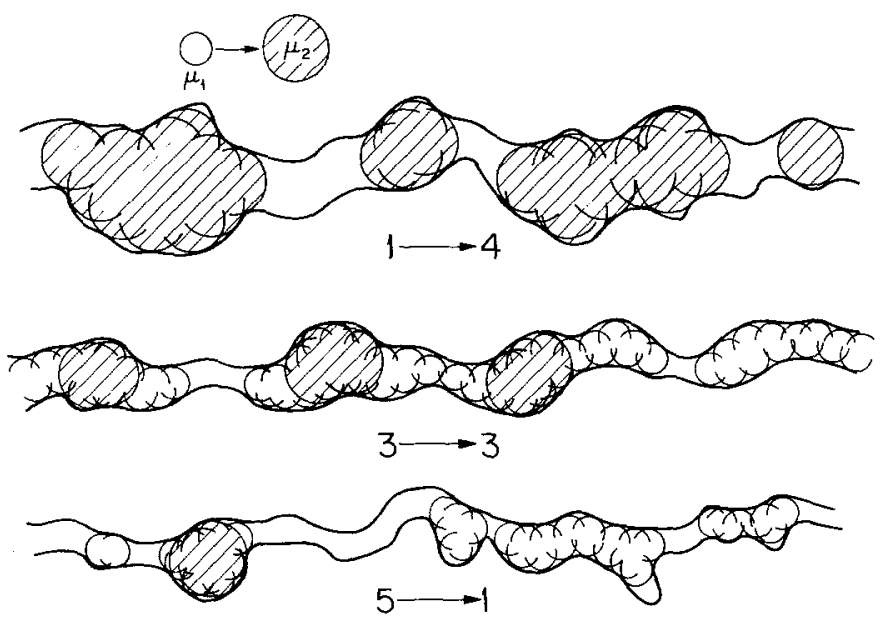

Fig. 4. Fragmentation and destruction of cave space when modulus is increased from $\mu_{1}$ to $\mu_{2}$. Top: one fragment becomes four; middle: three fragments become three; bottom: five fragments become one.

\section{THE LINKED MODULAR ELEMENT MODEL}

A construction that can be used to define cave length and volume (Fig. 5) is to fill it with touching modular elements of sizes $\eta>\mu$ (cave modulus $=$ $\mu$ ). Each modular element is as large as the walls of the cave will permit. Spheres are used for modular elements (Fig. 5), but any shape could be chosen. They are drawn sequentially, touching the one before and two points on caves walls.

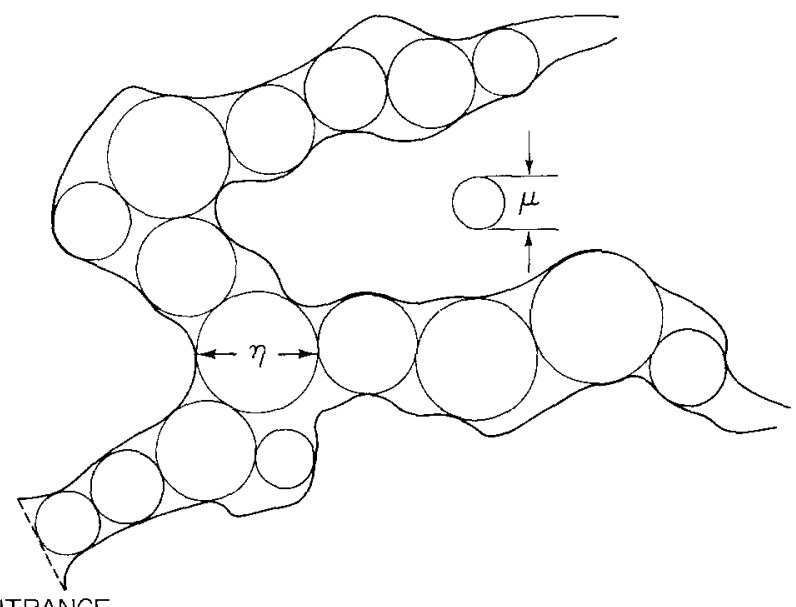

ENTRANCE

Fig. 5. Cave space of modulus $\mu$ filled with linked modular elements $\eta>\mu$. 
A question arises whether residual empty spaces between modular elements, or between modular elements and wall, should be filled with additional modular elements (so long as they are larger than $\mu$ ). Usually this is not done unless another passage or a passage termination is involved. This is consistent with the nature of surveyed cave length and minimizes accidental mismatches between what is perceived as a (proper) cave passage and what an arbitrary shape (e.g., sphere) would define, but a subjective element remains. This "problem" will be left for future resolution.

Construction locally is not unique. Successive sizes of modular elements will depend upon where one starts construction infilling. A particular construction, however, may be thought of as one manifestation of a statistical ensemble. Distributions and expected values to be derived are ensemble properties.

Define $M(\eta)$ to be the number of modular elements of size $\eta$ or larger in a region. Its density distribution is defined by

$$
m(\eta)=-d M(\eta) / d \eta
$$

The length of a cave will be defined as the sum of sizes of modular elements in that cave. Total length of all caves of modulus $\mu$ in the region therefore is given by

$$
L(\mu)=\int_{\mu}^{\infty} \eta m(\eta) d \eta=\int_{\mu}^{\infty} \ln (l \mid \mu) d l
$$

The right-hand integral is the same as eq. 6 , but here the relation between integrals allows calculating $m(\eta)$ from $n\left(l_{0} / \mu\right)$ Differentiating both integrals with respect to $\mu$ gives

$$
-\mu m(\mu)=\int_{\mu}^{\infty} l \frac{\partial n(l \mid \mu)}{\partial \mu} d l-\mu n(\mu \mid \mu)
$$

Substituting $N\left(l_{0} \mid \mu\right)$ from eq. 10 into Eq. 4 gives

$$
\begin{aligned}
n(l \mid \mu) & =\frac{\nu N_{\mathrm{p}}}{\mu_{\mathrm{p}}}\left(\frac{\mu}{\mu_{\mathrm{p}}}\right)^{\nu-\beta}\left(\frac{l}{\mu_{\mathrm{p}}}\right)^{-\nu-1} \quad \mu<l \quad \text { where } \\
N_{\mathrm{p}}=N\left(\mu_{\mathrm{p}} \mid \mu_{\mathrm{p}}\right) & =l_{0}^{\nu} N\left(l_{\mathrm{o}} \mid \mu_{\mathrm{p}}\right) / \mu_{\mathrm{p}}^{\nu}
\end{aligned}
$$

the total number of proper caves in a region. With eq. 14 substituted into eq. 13 and the integral evaluated, $m(\eta)$ is obtained as

$$
m(\eta)=\frac{\nu(\beta-1) N_{\mathrm{p}}}{(\nu-1) \mu_{\mathrm{p}}}\left(\frac{\eta}{\mu_{\mathrm{p}}}\right)^{-\beta-1} \quad 0<\eta
$$

and $M(\eta)$ as

$$
M(\eta)=\frac{\nu(\beta-1) N_{\mathrm{p}}}{(\nu-1) \beta}\left(\frac{\eta}{\mu_{\mathrm{p}}}\right)^{-\beta} \quad 0<\eta
$$


Ratio of number of modular elements larger than $\mu_{\mathrm{p}}$ in a region to number of proper caves in the same region is

$$
\frac{M\left(\mu_{\mathrm{p}}\right)}{N_{\mathrm{p}}}=\frac{\nu(\beta-1)}{(\nu-1) \beta}>1
$$

which must be greater than 1.0 because some caves are known to have more than one modular element. This gives

$$
\nu<\beta
$$

and establishes that more caves are created than lost by connections as $\mu$ decreases. This is the expected result for fractals, where greater fragmentation is expected as defining length decreases.

Total volume of caves in a region may be defined as total volume of modular elements, which is proportional to

$$
V=\int_{0}^{\infty} \eta^{3} m(\eta) d \eta=\frac{\nu(\beta-1) \mu_{\mathrm{p}}^{\beta} N_{\mathrm{p}}}{(\nu-1)(3-\beta)}\left[\eta^{-\beta+3}\right]_{0}^{\infty}
$$

The factor $\pi / 6$ (for spheres) has been omitted because no basis for choosing a particular shape for the modular elements for defining volume is recognized. The total volume contributed by the lower limit as $\eta>0$ must be finite, which requires $\beta<3$. This, however, gives infinite volume from the upper limit. However, the upper limit is constrained by the thickness of the strata in which caves occur, so distribution $m(\eta)$ must truncate at a large $\eta$. All fractal objects considered by Mandelbrot (1983) have such an outer cutoff at large $\eta$. This reasoning is different from that limiting $\nu$ in $N(l \mid \nu)$, because there length had no inherent associated volume.

Total area of caves in a region may be defined as total area of modular elements, which is

$$
A=\int_{0}^{\infty} \eta^{2} m(\eta) d \eta=\frac{\nu(\beta-1) \mu_{\mathrm{p}}^{\beta} \mathrm{N}_{\mathrm{p}}}{(\nu-1)(2-\beta)}\left[\eta^{-\beta+2}\right]_{0}^{\infty}
$$

A factor $\pi$ (for spheres) is omitted for the same reason as given for volume. By analogy to other fractally fragmented solids (Mandelbrot, 1983), the area contributed by small modular elements as $\eta \rightarrow 0$ is expected to approach infinity. Therefore, $\beta>2$ and

$$
2<\beta<3
$$

which is consistent with eq. 19 and the observed range of $\nu$. This states, in effect, that cave volume comes mostly from large modular elements and cave area comes mostly from small modular elements. A fractal dimension between 2 and 3 is characteristic of a fractally fragmented three-dimensional object (Mandelbrot, 1983). 


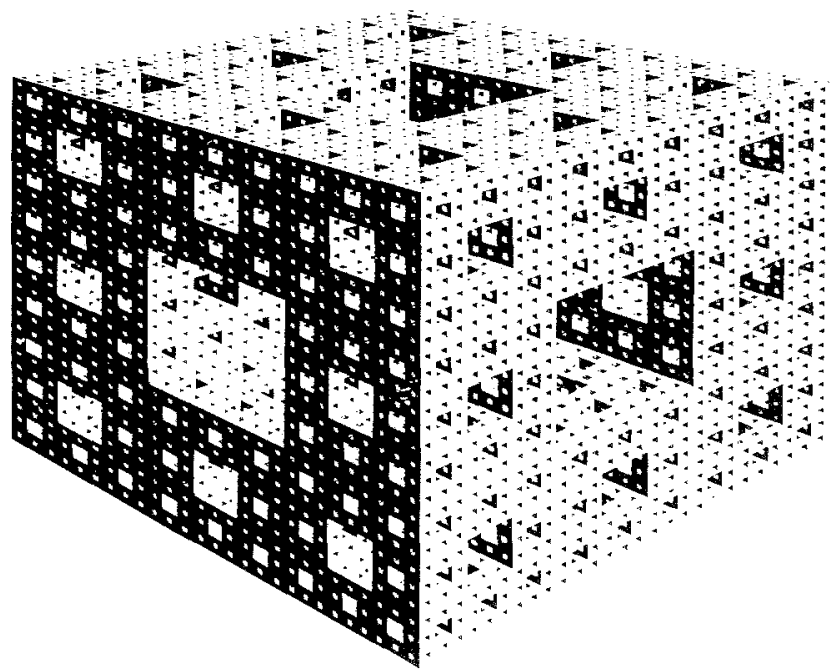

Fig. 6. Menger Sponge, a 2.7268 dimensional fractal solid. See Blumenthal and Menger (1970) and Mandelbrot (1983) for its construction. Reproduced with permission. Copyright 1983 by W. H. Freeman \& Co. All rights reserved.

These results tell us that modular elements can be considered as natural fractals of dimension $\beta$, the same as the dimension of total cave (fractal) length $L(\mu)$. Distribution $M(\eta)$ is not, however, practicably measurable. Only a few caves have entrances, and any measurements of the distribution of sizes of modular elements is conditional upon the properties of observable caves.

The Menger Sponge (Fig. 6) is a fractal object with $\beta=2.7268$ in the same range as caves. In the limit $\eta \rightarrow 0$ it has finite volume and infinite area and length. It exhibits the cave property of increasing length with decreasing modulus, but does not fragment also. It has an obvious outer cutoff. A fractal geometric object that resembled caves more closely would have to have random fragmentation, interconnections, and internal obstructions.

\section{CONDITIONAL MODULAR ELEMENT DISTRIBUTION}

Define $Q(\eta \mid l, \mu)$ as expected number of modular elements larger than $\eta$ in a cave of length $l$, modulus $\mu, \mu<\eta<l$. The contribution from all caves with $\eta<l$ gives the total number of modular elements larger than $\eta$ in a region. Thus

$$
\int_{\eta}^{\infty} Q(\eta \mid l, \mu) n(l \mid \mu) d l=M(\eta) \quad \mu<\eta
$$


Parameter $\mu$ cancels in the integrand because $M(\eta)$ is not a function of $\mu$, except for the trivial constraint that $\eta>\mu$. With $n(l \mid \mu)$ having the form of eq. 14 and $M(\eta)$ the form of eq. $17, Q(\eta \mid l, \mu)$ necessarily must have the form

$$
Q(\eta \mid l, \mu)=a \eta^{b} \mu^{c} l^{\gamma}
$$

Substituting this into 23 , integrating, and equating exponents of like terms, gives

$$
Q(\eta \mid l, \mu)=\frac{(\beta-1)(\nu-\gamma)}{\beta(\nu-1)}\left(\frac{\eta}{l}\right)^{-\gamma}\left(\frac{\eta}{\mu}\right)^{-\beta+\nu} \quad \mu<\eta
$$

A cave of length $\mu$, modulus $\mu$, has one modular element of size $\mu$. That is, $Q(\mu \mid \mu, \mu)=1$, so

$$
\begin{aligned}
\frac{(\beta-1)(\nu-\gamma)}{\beta(\nu-1)} & =1 \quad \text { or } \\
\gamma & =\frac{\beta-\nu}{\beta-1} \quad \text { and } \\
Q(\eta \mid l, \mu) & =\left(\frac{\eta}{l}\right)^{-\gamma}\left(\frac{\eta}{\mu}\right)^{-\beta+\nu}
\end{aligned}
$$

This expected conditional distribution of modular element sizes in a cave of length $l$ modulus $\mu$ represents a natural fractal of dimension $\chi=\gamma+\beta-\nu$ or, with eq. 27

$$
\chi=\frac{\beta(\beta-\nu)}{\beta-1}
$$

Parameter $\gamma$ is, incidentally, not a fractal dimension, because nothing is distributed hyperbolically with just that exponent.

Constraints already deduced for $\beta[2,3]$ and observed for $\nu[1.2,1.6]$ constrain $\gamma$ to $[0.4,0.9]$ and $\chi$ to $[0.8,2.7]$. Most important, samples of $Q(\eta \mid l$, $\mu)$ are measurable in known caves.

\section{MEASURING THE CONDITIONAL FRACTAL DIMENSION}

Cave survey data with passage widths and heights were obtained in computer-readable form for Little Brush Creek Cave, Utah (Halleck, 1984). The modular element measuring algorithm (Fig. 7) defines the $j$ th survey traverse from station $j$ to station $j+1$ having a distance $\lambda_{j}$. The lesser of passage width or height constrains exploration and survey and therefore this is taken to be the modular element diameter for station $j, \eta_{j, 1}$. Modular elements are interpolated between stations. End points of diameters $\eta_{j, 1}$ and $\eta_{(j+1), 1}$, at right angles to $\lambda_{j}$, are connected with straight lines representing nominal "walls." Intermedi- 


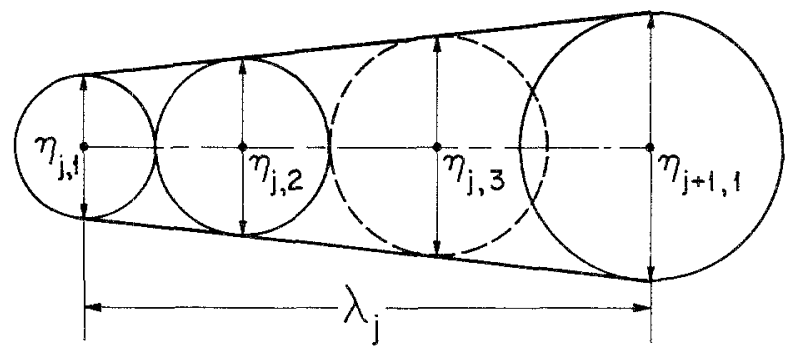

Fig. 7. Algorithm for linear interpolation of modular element sizes between stations of a cave survey.

ate modular elements of diameter $\eta_{j, k}$ are constructed along $\lambda_{j}$ so that they are touching, and ends of their diameters lie on the "walls." It follows that

$$
\begin{aligned}
\eta_{j, k} & =\left(\frac{1+\alpha_{j}}{1-\alpha_{j}}\right)^{k-1} \eta_{j, 1} \quad k=1,2, \ldots, n \quad \text { where } \\
\alpha_{j} & =\left(\eta_{j+1,1}-\eta_{j, 1}\right) / 2 \lambda_{j}
\end{aligned}
$$

The $n$th interpolated element has its center short of the $(j+1)$ th station. Then for $k=n+1$ [the $(j+1)$ th station]

$$
\frac{\eta_{j+1,1}}{\eta_{j, 1}}=\left(\frac{1+\alpha_{j}}{1-\alpha_{j}}\right)^{n}
$$

from which $n$ can be calculated as

$$
n=\ln \left(\frac{\eta_{j+1,1}}{\eta_{j, 1}}\right) / \ln \left(\frac{1+\alpha_{j}}{1-\alpha_{j}}\right)
$$

Equation 33 generally gives a noninteger number of modular elements in a traverse. This is not a problem: consider the example shown (Table 1) based on $\lambda_{1}=120$ (units), $\eta_{1,1}=32$, and $\eta_{2,1}=56$. Equation 33 gives $n=2.789$. The traverse contains two whole modular elements of size $32,39.1$, and 0.789 of a modular element of size 47.8 . Fractional elements cause no difficulty in

Table 1. Modular Element Interpolation Example

\begin{tabular}{lllc}
\hline$j$ & $k$ & $\eta_{j . k}$ & Count \\
\hline 1 & 1 & 32 & 1 \\
1 & 2 & 39.1 & 1 \\
1 & 3 & 47.8 & 0.789 \\
2 & 1 & 56 & 1 \\
\hline
\end{tabular}




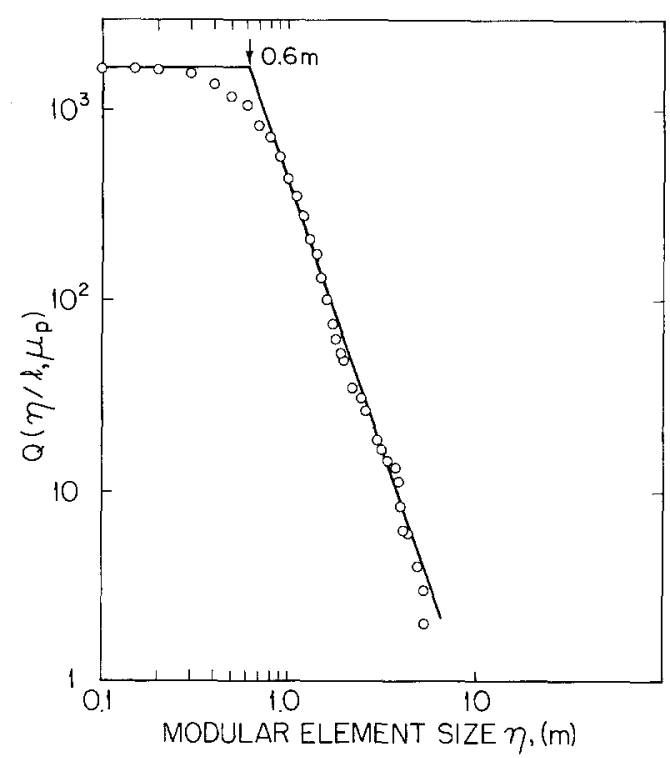

Fig. 8. Distribution of sizes of linked modular elements from Little Brush Creek Cave, Utah (not all data shown).

summing the cumulative distribution. This procedure also is consistent for $\lambda_{j}$ $<\eta_{j, 1}$.

Survey of the cave provided 562 traverses, of which 387 had height/width data at both stations. These produced 1651.9 modular elements. Their distribution (Fig. 8) is shown by only a portion of the data. The asymptotic slope for large $\eta$ is $\chi=2.79$ (this also tempts one to make an analogy to the Menger Sponge, whose dimension is 2.73 ). The inner-cutoff is near $\eta=0.6 \mathrm{~m}$, presumably close to the proper modulus $\mu_{p}$, although data were collected to $0.1 \mathrm{~m}$ (clearly not in proper cave). Equation 28 and assumptions of the linked modular element model, appear to be justified. This estimate of $\chi$ is from just one cave and may be different from the expected value for all caves in the region.

Fractal dimension $\nu$ has not been determined for this region of Utah. Thus, $\beta$ and $\gamma$ have been calculated for various values of $\nu$ (from eq. 29 with $\chi=$ 2.79; Table 2).

The fractal dimension of modular elements appears to be close to 3 , but uncertainty in $\nu$ and $\chi$ precludes precise estimation. Even though data (Fig. 8) suggests that eq. 28 is applicable, data were not collected with this application in mind. Sources of uncertainty include: the cave had not been completely surveyed; not all traverses had recorded station height/width; stations in narrow passages often are placed at convenient "wide" spots, losing intermediate smaller modular elements; and traverses crossing "rooms" may have stations 
Table 2. Alternative Fractal Dimensions for Utah

\begin{tabular}{ccc}
\hline$v$ & $\beta$ & $\gamma$ \\
\hline 1.0 & 2.79 & 1.00 \\
1.1 & 2.94 & 0.95 \\
1.2 & 3.09 & 0.90 \\
1.3 & $>3$ & 0.87 \\
\hline
\end{tabular}

with smaller height/width than intermediate points, losing intermediate larger modular elements. The last two problems also are failures for linear interpolation (Fig. 7).

\section{APPLICATIONS}

\section{Defining Cave Length and Volume}

The linked modular element model and the algorithm for counting modular elements between stations, provides a logical definition of "length of a cave." Some controversy about this concept presently exists, especially among those contending with having surveyed the "longest cave" (Chabert and Watson, 1981). Questions that arise are: Should width of a large room be included as well as its length? (no). Is extra length being measured when several traverses start at the same station? (yes). Should passages on both sides of a rock pillar in the middle of a larger passage be measured separately? (yes). Modular-element answers are indicated in parentheses. Dubljanski, Iljuhin, and Lobanov (1980), and Šušteršič $(1978,1980)$, also discuss these issues.

Sušteršič (1980) suggested another objective measure of cave length-the path length of the center of a sphere that moves "smoothly" through the cave, changing diameter to remain always in contact with walls. However, no simple way to derive this measure from survey data is known. In addition, irregularity (fractal?) of walls will make the path of the center also irregular (fractally?) and the resulting length may be subject to Richardson's Law-it will depend upon the scale of the measuring stick used. The linked modular element model does not have this difficulty.

Cave volume is defined also by the model. For example, estimated total cave volumes in Pennsylvania are calculated (Table 3) for different ranges of $\eta$ from eq. 20 with finite limits. Parameters used are $\nu=1.4, \beta=2.79, \mu_{p}=$ $0.5 \mathrm{~m}$, and $N_{p}=3.0 \mathrm{E}+05$.

Numerical results are sensitive to $\beta$, but the general implication is that a considerable cave volume exists at small $\eta$. The range $\eta=0$ to $0.0001 \mathrm{~m}$ is 
Table 3. Cave Volumes in Pennsylvania in Different Modular Element Size Ranges

\begin{tabular}{|c|c|}
\hline$\eta$ range $(\mathrm{m})$ & volume $\left(\mathrm{m}^{3}\right)$ \\
\hline-0.0001 & $1.1 \mathrm{E}+06$ \\
\hline $0.0001-0.01$ & $1.8 \mathrm{E}+06$ \\
\hline $0.1-1.0$ & $4.8 \mathrm{E}+06$ \\
\hline-100 & $1.3 \mathrm{E}+07$ \\
\hline
\end{tabular}

secondary "porosity" inherent to the rock and present in cracks. Of course, $\beta$ possibly may change in the range as it does for microcraters (Morisson and Clanton, 1979).

\section{Survey Traverses in a Cave}

For $\eta=\mu=\mu_{p}$, eq. 28 becomes

$$
Q_{p}=Q\left(\mu_{p} \mid \ell, \mu_{p}\right)=\left(\ell / \mu_{p}\right)^{\gamma}
$$

which gives the expected number of modular elements in a proper cave. Each traverse in a survey can cover at least two, and likely three, modular elements. Therefore, an estimate of the upper limit on number of traverses required to survey a cave is $Q_{p} / 3$. Values for different cave lengths (Table 4) were estimated using $\mu_{p}=0.6 \mathrm{~m}$ and $\gamma=0.9$ from Table 2 .

The implication is that this number does not increase as rapidly as the length of a cave because of the increasing expectation of larger modular elements. Brush Creek Cave (Utah), with a surveyed length of $3380 \mathrm{~m}$, is expected to have fewer than 791 traverses (stations), whereas 562 were employed.

\section{Subjective Concept of Cave Geometry}

a. Draw a "typical" cave that reflects your concept of cave geometry. Include in the drawing passages that limit exploration, i.e., down to $\mu_{p}$ (an example is Fig. 5).

Table 4. Maximum Number of Traverses in Caves of Different Lengths (Modulus $0.6 \mathrm{~m}$ )

\begin{tabular}{cr}
$l(\mathrm{~m})$ & \multicolumn{1}{c}{$Q_{p} / 3$} \\
\hline $1 . \mathrm{E}+02$ & 33 \\
$1 . \mathrm{E}+03$ & 265 \\
$1 . \mathrm{E}+04$ & 2100 \\
$1 . \mathrm{E}+05$ & 16700 \\
\hline
\end{tabular}


b. Fill your cave with modular elements (as in Fig. 5).

c. Measure the length of your cave as the sum of diameters of modular elements, using $\mu_{p}$ as the "unit" of length. (Figure 5 has a cave length of $l / \mu_{p}$ $=27.1$.)

d. The expected number of modular elements (Fig. 5) is

$$
Q_{p}=\left(/ / \mu_{p}\right)^{0.9}=19.5
$$

Cave "Fig. 5" has 16 modular elements-intriguingly close. This "parlor trick" has been found to appeal to speleologists. It appears to have deeper implications (related to volumetric irregularity, or "lumpiness", of caves), which have yet to be explored.

\section{TWO-DIMENSIONAL CAVES}

The foregoing analysis is easier to illustrate with two-dimensional figures (plan projections) than with three-dimensional figures. In fact, "real" caves present many quandries about procedures, which are relatively natural for twodimensional cave drawings. For example, should tall, narrow (low, wide) passages be filled to the ceiling (walls) with layers (rows) of modular elements? Lengths of such passages would then become enormous but not reflect survey practices that normally obtain data (Figs. 1 and 8). Should, then, some other explorer shape be used to define the passage and fill it with modular elements? These questions do not have clear answers yet, but apparently an anthropomorphic explorer is an ill-defined entity. Despite this, data that are obtained exhibit great regularity, which has been illustrated. Answers to this puzzle may come from philosophy as likely as from geography.

All of the previous analysis is repeated readily for two-dimensional ("Flatland") caves. Total length (still defined as sum of modular element diameters) may go to infinity, but area must be finite. This still requires that $\nu>1.0$ (from eq. 5). However, $1.0<\beta<2.0$ (from eq. 21). These alone constrain $\gamma<$ 1.0 and $\chi<2.0$. If $\nu$ is constrained, as before, to $[1.2,1.6]$ (very speculative!), then $\gamma$ is constrained to $[0,0.8]$ and $\chi$ to $[0,1.6]$. These are too broad to reach clear conclusions, except for the previous "parlor trick," which after all was proposed for a two-dimensional cave construction. The exponent should be less than 0.8 . If 0.8 is used as the exponent for calculating expected number of modular elements (Fig. 5), $Q_{p}=14.0$, even closer to the 16 observed. The reader may experiment with this.

\section{DISCUSSION}

This work has attempted to take some first steps from observation of hyperbolically distributed cave lengths, toward a general but quantitative descrip- 
tion of cave geometry. Tenets are acceptance of caves as natural fractals, and use of self-similar properties of fractals as bases for postulating a linked modular element model for cave geometric properties. This is, at best, a still crude abstraction of cave geometry. For one thing, only limited data are available despite the large number of mapped caves. Cave surveying is difficult work, and most has been done by voluntary effort. Because data about cave geometry has little use, except for distances and orientations, little more has been recorded, except to draw approximate passage shapes. However, what the most useful abstractions of the actual complexity are for describing or predicting cave features is unclear. Hopefully, the modular element model will stimulate future insights into this question.

Distribution $M(\eta)$ is a marginal distribution and $Q(\eta \mid l, \mu)$ remains only a marginal distribution, conditional on a local measure $l$. The relation between $M(\eta)$ and $N(l \mid \mu)$ from eqs. 13-17 applies to hyperbolically distributed "beads in a box." No arrangement is implied. Even the joint distribution of successive modular element $\eta_{j}$ is unstudied. This pattern would constitute a stochastic process of successive $\eta_{j}$ and orientations. If more data are gathered within the framework of the linked modular element model, patterns may be analyzed. Eventually, all that and more will need to be imbedded in a geological framework, with connections to processes.

What is the reason that cave geometry and many other natural fractals appear to be self-similar? A first guess is that the variety of geomorphic processes occurring in a complex geological setting, each of which has some related characteristic lengths, in totality introduce so many different characteristic lengths over a wide range, that none dominate. Something is acting here akin to the central limit theorem for the normal distribution but which leads to hyperbolic distributions in nature. This has not been clearly formulated, although it was recognized long ago that "two fundamental types of statistical distributions" seems to exist (Korčak, 1940).

At the very least, a fractal analysis of cave geometry works toward eliminating the present anthropomorphism of cave studies, where caves are defined frequently as only "enterable by humans," thereby implicitly limiting their study. If this work serves to alter that perspective, one aspect will have been useful.

\section{ACKNOWLEDGMENTS}

Cave survey data for Little Brush Creek Cave, Utah, were provided on computer tape by John B. Halleck. John H. Rosenfeld prepared the code for the modular-element algorithm and carried out the data analysis. Mr. Rosenfeld was supported in part by the College Work-Study Program with support from the Rackham School of Graduate Studies at the University of Michigan. Permission to reproduce Fig. 1 was given by The Journal of Geology (University 
of Chicago), and for Fig. 6 from Mandelbrot (1983), by W. H. Freeman and Company.

\section{REFERENCES}

BlumenthaI, L. M. and Menger, K., 1970, Studies in geometry: W. H. Freeman, San Francisco, p. 502.

Chabert, C. and Watson, R. A., 1981, Mapping and measuring caves-a conceptual analysis: Bull. Nat. Speleo. Soc., v. 43, p. 3-11.

Curl, R. L., 1960, Stochastic models of cavern development: Bull. Nat. Speleo. Soc., v. 22, p. $66-76$.

Curl, R. L., 1964, On the definition of a cave: Bull. Nat. Speleo. Soc., v. 26, p. 1-6.

Curl, R. L., 1966, Caves as a measure of karst: J. Geol., v. 74, no. 5, part 2, p. 798-830.

Dubljanski, V. N., Iljuhin, V. V., and Lobanov, J. E., 1980, Some problems relating to the morphometry of karst caves: Nase Jame, v. 21, p. 75-84.

Fréchet, M., 1941, Sur la loi de répartition de certaines grandeurs géographiques: J. Soc. Stat. Paris, v. 82 , p. $114-122$.

Halleck, J. B., 1984, personal communication.

Korčak, J., 1940, Deux types fondamentaux de distribution statistique: Bull. Inst. Int. Stat., v. 30. p. $295-299$.

Mandelbrot, B. B., 1983, The fractal geometry of nature: W. H. Freeman, San Francisco, 468 p.

Morrison, D. A. and Clanton, U. S., 1979, Properties of microcraters and cosmic dust of less than 1000 A dimensions: Proceedings of the 10th Lunar Planetary Science Conference, p. 16491663.

Richardson, L. F., 1961, The problem of contiguity: An appendix of statistics of deadly quarrels: Gen. Sys. Yearbook, v. 6, p. 139-187.

Šušteršič, F., 1978, What is speleometry?: Nase Jame, v. 20, p. 21-29.

Šušteršič, F., 1980, Some basic dimensions of the speleogenes: Nase Jame, v. 21, p. 61-73. 